\title{
A minicomputer system for recording the dynamic properties of individual operant responses*
}

\author{
STEPHEN C. FOWLER $\dagger$ \\ University of Mississippi, University, Mississippi 38677
}

\begin{abstract}
A PDP-12 is used to conduct operant experiments in which peak force, duration, time integral of force, and interresponse time may each serve as the criterion response property for reinforcement, and all four properties are simultaneously recorded as dependent variables. Calibration, acquisition and control, and data analysis programs are described.
\end{abstract}

Conventional operant conditioning methodology defines a response as a discrete event registered by the closure of a microswitch. The typical laboratory instruments, regardless of whether they are computer-based, count these events and measure the time in tervals between them. This approach certainly has provided much valuable information. However, there is mounting evidence that limiting operant analysis to an examination of the time between switch closures is tantamount to ignoring important behavioral information residing in the amplitude and duration of the individual responses (Notterman \& Mintz, 1965; Weiss, 1973).

Several commercially available computer-based systems for on-line data acquisition and control have been developed for the case where a response is viewed as a ballistic event. Grason-Stadler's SCAT, Lehigh Valley's ACT, and Digital Equipment Corporation's (DEC) SKED, all assume that the response will reach the computer in digital form (see Millenson, Kehoe, Tait, \& Gormezano, 1973). Yet, the problems posed by processing the voltage analogs of force for individual responses has not reached such a stage of solution. The purpose of this paper is to describe a computer system-both hardware and software-designed for conducting an operant analysis in which peak force, duration, and time integral of force of individual responses assume a position of importance equal to that of interresponse time.

\section{HARDWARE}

A block diagram of the system is presented in Fig. 1. As shown, the minimum PDP-12 (DEC) configuration required includes $4 \mathrm{~K}$ of core, two TU55 magnetic tape transports with tape control, AD-12 analog-to-digital converter, KW12 real-time clock, relay buffer, and

\footnotetext{
*Preparation of this paper and development of the described system were supported by NIMH Grant 18189 , J. M. Notterman, Principal Investigator. Xenia Coulter wrote the programs PDERMS and XFREQ, and William C. Libby prepared the FOCAL programs.

†This work was carried out while the author was at Princeton University, Department of Psychology.
}

ASR-33 Teletype. Instead of the conventional microswitch manipulanda, the system uses Hewlett-Packard microforce transducers (FTA-100, resonant frequency of $390 \mathrm{~Hz}$ ) in conjunction with Hewlett-Packard transducer amplifier indicators (311-A). The latter item is compatible with most strain-gauge and differential transformer transducers. Low-pass $(50 \mathrm{~Hz}$ and below) filters are included to reduce the effects of extraneous electrical transients associated with operation of other equipment in the laboratory.

Behavioral equipment includes two Skinner boxes (23 cm long $\times 20.5 \mathrm{~cm}$ wide $\times 19 \mathrm{~cm}$ high), each being enclosed in a sound-attenuating cubicle equipped with houselight, pellet dispenser, and exhaust fan. Each manipulandum is positioned outside the box $(\sim 3 \mathrm{~cm}$ from an opening in the cage wall to the center of the manipulandum). The portion of the transducer available to the organism is an 18-mm-diam disk; the surface of the disk is parallel to the horizontal plane and $60 \mathrm{~mm}$ above the grid floor. Thus, forces are exerted on the manipulandum by a downward pressure of the rat's paw. Since excursion of the disk is less than $0.4 \mathrm{~mm}$ for forces up to $200 \mathrm{~g}$, the manipulandum is essentially isometric.

\section{FOUR RESPONSE MEASUREMENTS}

Following the lead of Notterman and Mintz (1965), the present system defines a response in terms of a "threshold" force (see Fig. 2). Any force above threshold will initiate program loops for recording and measuring the response. The threshold is usually set just high enough to prevent electrical noise from registering as a response. The system records peak force, duration, time integral of force, and interresponse time for each response. Peak force $\left(F_{p}\right)$ is simply the maximum force amplitude attained by a response. As shown in Fig. 2, the duration is the amount of time that the force remains above threshold. Likewise, the time integral of force ( $\mathrm{Fdt}$, or effort) is the area under the force-time curve measured while the force is above threshold. Interresponse time (IRT) is marked off by the force of the preceding response falling below threshold and the 


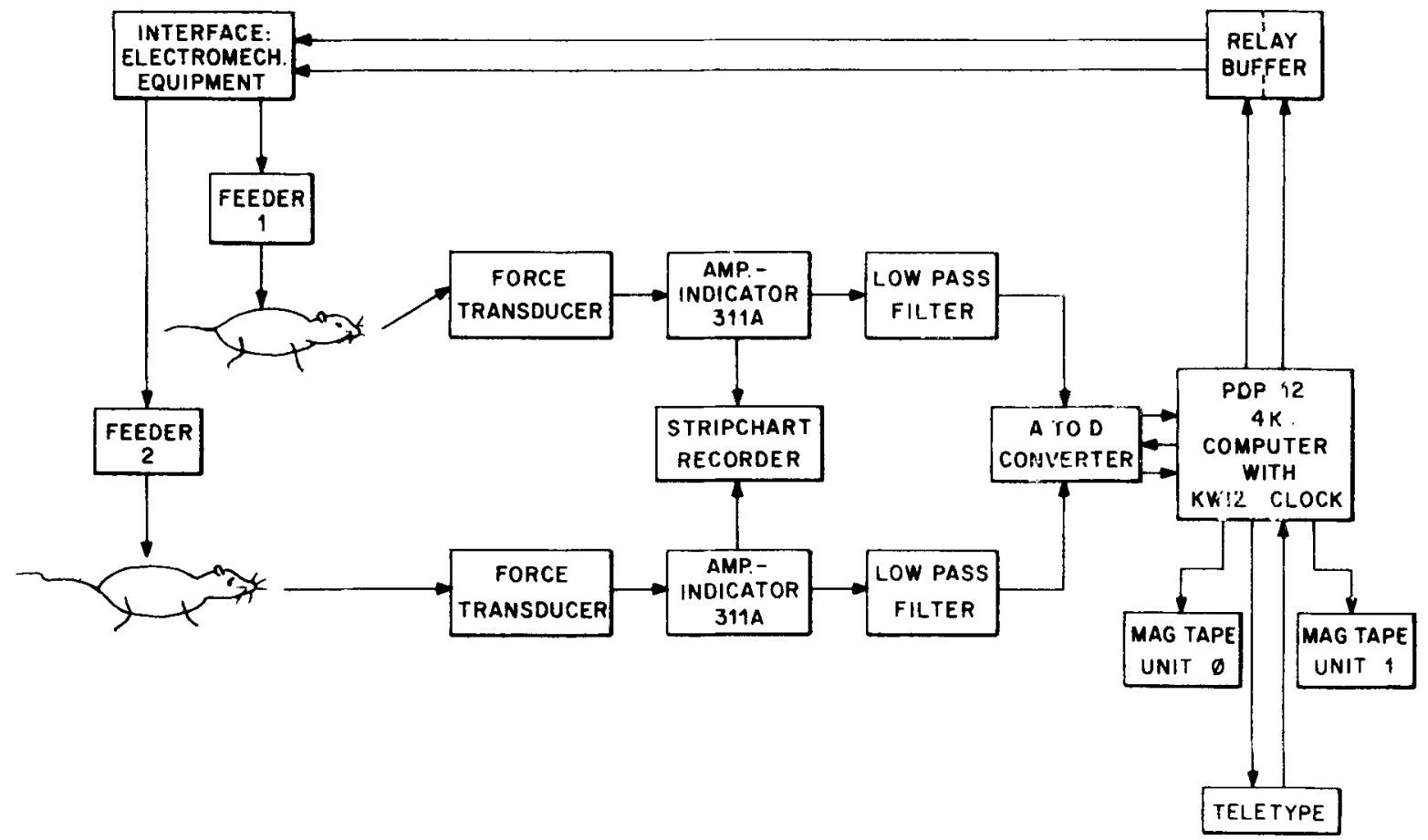

Fig. 1. Block diagram representing the components of a PDP-12-based system for recording the dynamic properties of operant responses.

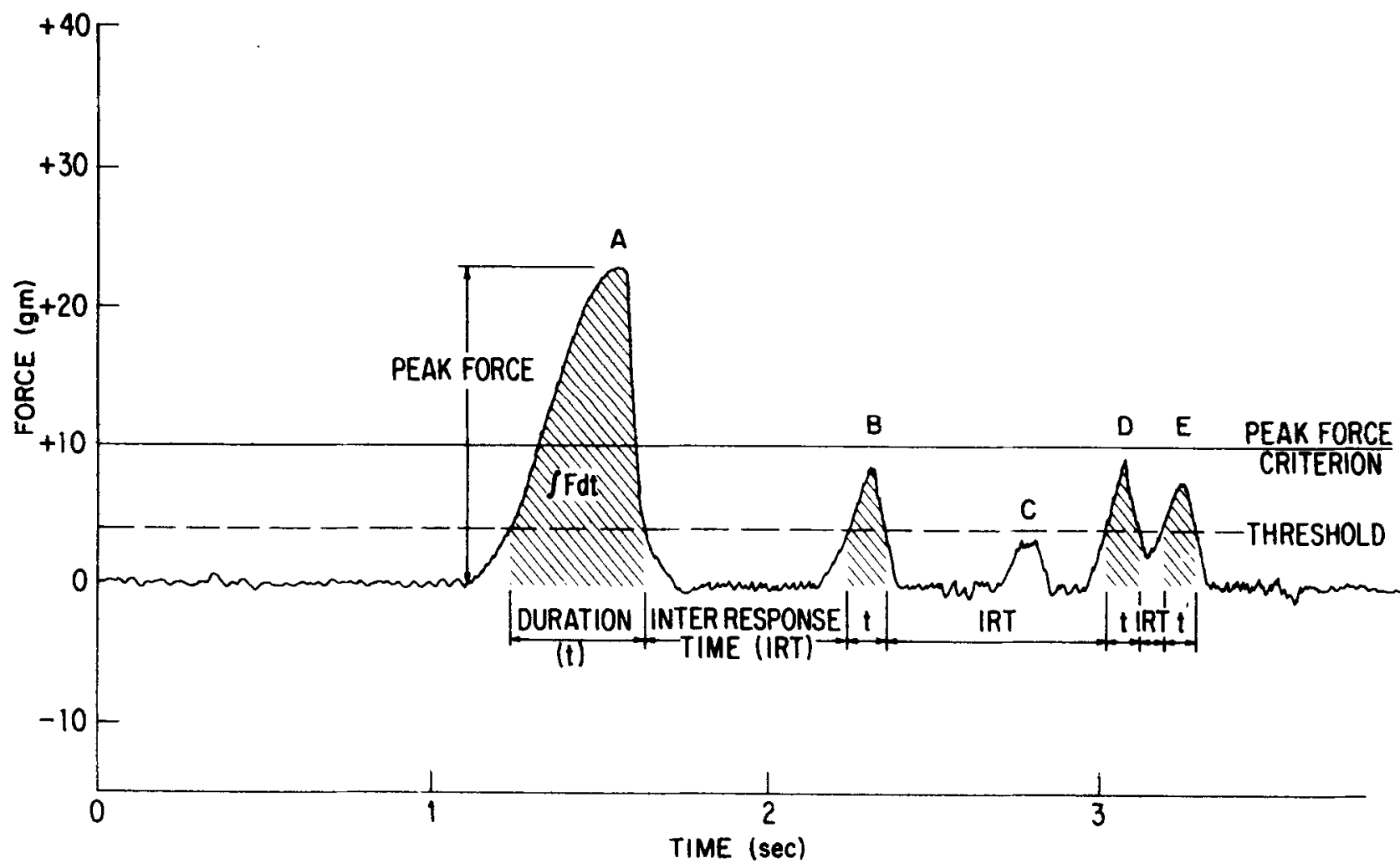

Fig. 2. Schematic representation of a force-time record showing peak force, duration, time integral of force, and interresponse time. A, B, D, and E are registered as separate responses. Response $A$ reached the peak force reinforcement criterion of $10 \mathrm{~g}$; Responses $B, D$, and $E$ were of insufficient force to meet the criterion and were not reinforced. $C$ was not recorded at all, as the force failed to reach the threshold of $4 \mathrm{~g}$ (adapted from Notterman \& Mintz, 1965). 
succeeding response force rising above threshold. Thus, IRT is assumed to be a response property that precedes the response (cf. Kramer \& Rilling, 1970).

The "criterion" is the requirement that must be met (or exceeded) before programmed consequences are presented to the organism. For instance, a $10-\mathrm{g} \mathrm{Fp}$ criterion for reinforcement by a food pellet is illustrated in Fig. 2. In general, the criterion may be specified in terms of Fp, $t, \int F d t$, or IRT.

In most applications, reinforcement is delivered upon response termination. This procedure ensures that reinforcement delivery cannot serve as an exteroceptive cue that the criterion value has been attained, and thereby increases the likelihood that the organism will attend to proprioceptive cues.

\section{SOFTWARE}

The general functions performed by the system are threefold: calibration, acquisition and control, and data analysis. Separate programs were written to accomplish each of these tasks. Except for some of the data analysis programs written in $4 \mathrm{~K}$ FOCAL, all the programs were composed in assembly language. Limitations of core, personnel, and financial resources made programming in a higher order language infeasible (cf. Pilla, 1973; Siegel, 1973).

\section{CALIBRATION}

The four response properties are defined in terms of force or time or both of these quantities. The time base is a crystal oscillator in the KW12 clock. Presumably calibration of the crystal is accomplished during its manufacture. However, the force transducer itself must be calibrated so as to yield convenient units of measurement. Calibration is also required to fix a zero origin when no load is on the transducer. How frequently this is necessary will, of course, depend upon the type of transducer being used. The differential transformer supplied by Hewlett-Packard (FTA-100) is remarkably resistant to drift. Thus, calibration may be required as infrequently as once per week. On the other hand, certain strain-gauge transducers, which have less stable zero points, may have to be adjusted daily.

The calibration program, CALIB, is a combination of assembly language and DEC's PDP-8 double-precision floating-point package. When used in conjunction with the 311-A amplifier-indicator, calibration is straightforward. The program repeatedly obtains $100_{10}$ voltage samples from Channel 10 of the A-D converter. The mean and standard deviation for the 100 samples are computed and then typed. The ubiquitous problem of electrical noise renders such a statistical treatment mandatory. With no load on the transducer, the 311-A controls are adjusted until a near-zero reading is achieved on the Teletype. Then known masses are placed on the transducer, and the controls on the 311-A are manipulated until convenient units of measurement are selected.

\section{ACQUISITION AND CONTROL}

Several programs are available. Each one is adapted to the requirements of the specific experimental questions under consideration. However, because they all share the same essential subroutines, the description of one program will serve to illustrate the general approach. This program, GENEXP, was used successfully in a series of experiments examining the relation between force proportional reinforcement and average rate of reinforcement (Fowler \& Notterman, in press). Thus, GENEXP is thoroughly debugged.

The program begins with a subroutine (ACCEPT), which requests from the Teletype keyboard the parameters that specify the criterion property and the type of reinforcement contingency. The criterion property may be Fp, $t, \int F d t$, or IRT. After the type of criterion is chosen, the kind of reinforcement contingency is selected from among three possible types: regular reinforcement, proportional reinforcement, or band reinforcement. With the regular reinforcement contingency, the specified response property must equal or exceed the criterion value before reinforcement will be forthcoming. For example, if IRT is the criterion response property, then the regular contingency is simply a differential reinforcement of low rate (DRL) schedule. Or if $\mathrm{Fp}$ is the criterion response property, regular reinforcement is equivalent to a CRF schedule for responses having Fps equal to or greater than the criterion; responses with $\mathrm{Fp}$ between threshold and criterion are recorded but go unreinforced. For the proportional contingency, the number, probability, quantity, or quality of reinforcements is delivered in proportion to the magnitude of the criterion response property (cf. Notterman \& Mintz, 1965; Fowler \& Notterman, in press). Thus, the proportional contingency permits the $E$ to reinforce specific quantitative subclasses of response. Finally, band contingencies may be selected so that the response measurement must fall within specified lower and upper limits before reinforcement is presented (cf. Notterman \& Mintz, 1965). In the case where IRT serves as the criterion property, the band contingency is equivalent to a DRL with limited hold.

After the criterion property and the type of reinforcement contingency are typed, the program repeatedly samples the voltages arriving from the two transducers. The sampling rate is $100 / \mathrm{sec}$. For experiments with rats, squirrel monkeys, or humans, this sampling rate is sufficiently rapid to reproduce, with satisfactory resolution, the waveforms of responses. Since these Ss possess hands or paws padded with inelastic fatty tissue, short duration, ballistic responses rarely have durations of less than $.08 \mathrm{sec}$. However, for more elastic collisions between organism and transducer, 
such as pigeon pecks, a higher sampling rate is required (Rilling, Kramer, \& Askew, 1970).

Every sample is subjected to a threshold test. If the voltage ( $\mathrm{Fp}$ ) is found to be above threshold, the program enters a response-in-progress loop which raises a sof tware response flag and calls the subroutine COLECT. This subroutine up-dates the $\mathrm{Fp}$ and $\mathrm{t}$ and computes $\int \mathrm{F} d \mathrm{t}$. Since $\mathrm{Fp}$ is the waveform maximum, it is obtained simply by comparing the current sample against the previously stored $\mathrm{Fp}$ value. If the current sample is larger, it replaces the previous Fp. Because COLECT is called once per $.01 \mathrm{sec}$ when a response is in progress, $t$ is recorded in units of $.01 \mathrm{sec}$ by tallying 1 in the appropriate memory register every pass through the subroutine.

When $\int F d t$ is recorded, the 12-bit word length of the PDP-12 requires a compromise between precision and overflow. The successive force values that occur during a response are added together in double precision to yicld $\int F d t$ in 100ths of gram-seconds. Upon response termination, $\int F d t$ is scaled down to one 12-bit word for eventual storage on magnetic tape. The amount of scaling is flexible and may be chosen according to the requirements of the particular experiment.

When the voltage falls below threshold and, at the same time, the response flag is raised, this conjunction of events signals the end of a response. The response flag is then lowered, and the subroutine STORE is called. This subroutine transfers the measurements $\left(\mathrm{Fp}, \mathrm{t}, \int \mathrm{Fdt}\right.$, IRT) to the data table, which, when filled, is written on magnetic tape. STORE calls the subroutine FINDRW, which computes the "reinforcement control word" according to the criterion and contingency specified previously by ACCEPT. The reinforcement control word is loaded into the relay buffer to energize appropriate relays to deliver reinforcement.

In the case where the voltage is below threshold and the response flag is not raised, STORE and COLECT are skipped and the IRT subroutine is called. Here IRT is recorded in double precision and is scaled down by STORE before being written on magnetic tape.

Every $.01 \mathrm{sec}$, the threshold test is made for each transducer and program flow is routed accordingly. Although both conditioning stations are subject to identical contingencies, they are virtually independent in operation. That is, a session may be initiated or terminated in one station without affecting the operation of the other. This feature is advantageous because quite often the length of a session will be contingent upon the organism's behavior. In one version of GENEXP, the independence of the two stations is achieved by means of a pair of sense switches. When set to 1 , the switches for Station 1 effectively cause all instructions pertaining to Station 1 to be skipped. Setting the sense switches also issues a write instruction to transfer the data remaining in core to magnetic tape. In addition, the data tape block number for the next session is printed, thereby providing a record of the location of data for each S. A new session in Station 1 is started by setting the pair of sense switches back to 0 .

GENEXP writes the data on magnetic tape in a format compatible with the data reduction programs described below. A separate data tape is used for each of the two conditioning stations.

\section{DATA REDUCTION}

In many of the previous experiments conducted with this sytem, three types of data summary have proven particularly useful. These are session means, standard deviations, and frequency distributions for each of the four dependent variables. Written in assembly language and combined with DEC's PDP-8 double-precision floating-point package, the programs PDERMS and XFREQ perform these routine data reduction tasks. Both programs read data from magnetic tape in the same format used by GENEXP.

The program PDERMS obtains the means and standard deviations for Fp, t, fFdt, and IRT on a session-by-session basis. The results, including the number of responses, are printed on the Teletype along with identifying tape block numbers. Several useful options are available. For example, one may have the statistics printed for successive fractions (Vincent blocks) of a session. The number of below-criterion responses may also be obtained. In most experiments, the output from PDERMS serves as the input data for a variety of inferential statistical programs written in FOCAL (4K). Currently, the FOCAL library includes programs for analysis of variance, $t$ test, correlation, and nonparametric tests.

Frequency distributions for the four response measurements are computed and printed by XFREQ. Both relative frequency and cumulative frequency distributions comprise 64 class intervals. The size of the class interval may be separately selected for each of the four variables.

Often, the $E$ feels the need to examine the data at a finer grain than that provided by frequency distributions and associated parameters. Graphical display, of course, is ideal for this purpose. Because the laboratory had neither an X-Y plotter nor a Polaroid camera for converting the PDP-12 scope display to hard copy, a program, PLOTCR, was written which permitted a cumulative recorder to be used as an output device driven by the relay buffer. Despite its extreme lack of speed, this method can produce a readable graphic reconstruction of $\mathrm{Fp}$ and IRT relations. An example is shown in Fig. 3. Each vertical line represents one response. The height of the line is proportional to Fp, and the horizontal distance between lines is proportional to IRT. This particular record is for a rat responding on a DRL $10-\mathrm{sec}$ schedule with a Fp requirement of $8 \mathrm{~g}$. Clearly, average response rate was relatively high at the beginning of the session, as indicated by the close spacing of vertical lines. Moreover, responses tend to be 
$40 \mathrm{GN} \quad$ DRL SEC $10 \mathrm{SEC}-8 \mathrm{GM}$

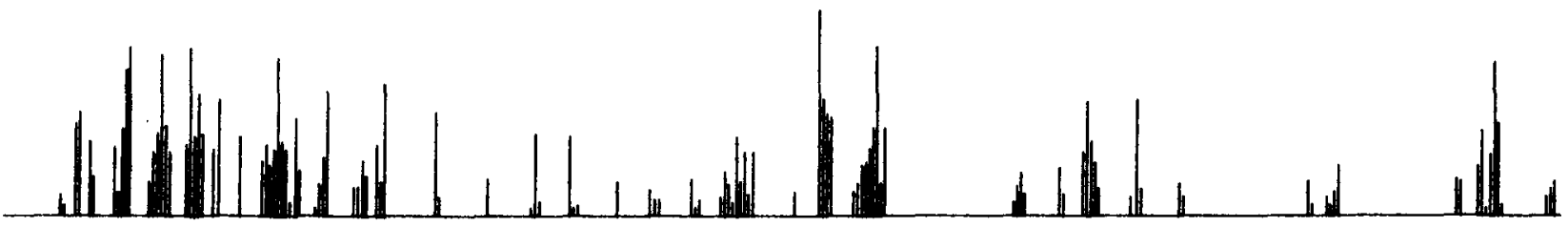

Fig. 3. Graphic display produced by a cumulative recorder under computer control. Each vertical line represents one response emitted by a rat during the initial portion of a session in which DRL $10 \mathrm{sec}$ and $8 \mathrm{~g}$ were the joint criteria for reinforcement. Height of vertical lines and distance between lines are proportional to Fp and IRT, respectively.

emitted in bursts. Even though this method of graphic output is quite wasteful of computer time, it can be useful when computer time is abundantly available and computer-oriented plotting hardware is not accessible.

\section{CONCLUSION}

Although the system was described in terms of animal learning paradigms, it has been used, with a few minor modifications, for investigating certain aspects of proprioceptive learning and memory in humans. Moreover, the addition of a few simple subroutines transforms the present system into an accurate and efficient means of implementing a number of psychophysical procedures, such as magnitude estimation and cross-modality matching, which often involve the emission of response force. Thus, the recording of individual response properties may be added to the growing list of requirements for a highly general software package that will accommodate the needs of a variety of experimental psychology laboratories.

\section{REFERENCES}

Fowler, S. C., \& Notterman, J, M. Reinforcement rate and force proportional reinforcement. Learning \& Motivation, in press. Kramer, T. J., \& Rilling, M. Differential reinforcement of low rates: A selective critique. Psychological Bulletin, 1970, 74, 225-254.

Millenson, J. R., Kehoe, E. J., Tait, R. W., \& Gormezano, I. A minicomputer program for control and data acquisition in classical conditioning. Behavior Research Methods \& Instrumentation, 1973, 5, 212-217.

Nottexman, J. M., \& Mintz, D. E. Dynamics of response. New York: Wiley, 1965 .

Pilla, M. A. Co-existing with on-line systems. Behavior Research Methods \& Instrumentation, 1973, 5, 80-82.

Rilling M. Kramer, T. J.\& Askew, H. R. A preliminary analysis of the dynamics of the pecking response in pigeons. Journal of the Experimental Analy sis of Behavior, 1970, 13, 267-278.

Siegel, W. Choosing a programming system for on-line control of research. Behavior Research Methods \& Instrumentation, $1973,5,131-133$.

Weiss, B. Can computers answer behavioral questions? Behavior Research Methods \& Instrumentation, 1973, 5, 67-79. 